\title{
Immer außer Atem? Eine Analyse der ärztlichen Tätigkeit in der Pneumologie
}

\author{
Always Out of Breath? An Analysis of a Doctor's Tasks in Pneumology
}

Autoren

Institute
S. Mache ${ }^{1,2}$, N. Jankowiak' ${ }^{1}$ C. Scutaru' ${ }^{1}$ D. A. Groneberg ${ }^{1}$

Institut für Arbeitsmedizin, Charité - Universitätsmedizin Berlin, Freie Universität und Humboldt Universität, Berlin

Klinik für Psychosomatik, Charité - Universitätsmedizin Berlin, Freie Universität and Humboldt Universität, Berlin eingereicht 16.3.2009

akzeptiert nach Revision 11. 5.2009

\section{Bibliografie}

DOI 10.1055/s-0029-1214798

Pneumologie 2009; 63:

369-373 @ Georg Thieme

Verlag KG Stuttgart · New York ISSN 0934-8387

\section{Korrespondenzadresse Stefanie Mache}

Institut für Arbeitsmedizin Charité - Universitätsmedizin Berlin

Humboldt-Universität zu Berlin und Freie Universität Berlin Thielallee 69-73

14195 Berlin

stefanie.mache@charite.de

\section{Zusammenfassung \\ $\nabla$}

Hintergrund: Derzeit ist die berufliche Situation der Ärzte in Deutschland durch einen zunehmenden ökonomischen Druck gekennzeichnet. Dieser spiegelt sich im medizinischen Vorgehen, in organisatorischen Abläufen und in der Arbeitszufriedenheit. Da diese Angaben nur subjektive Wahrnehmungen der Ärzte spiegeln, ist es zwingend notwendig, objektive Daten über die Arbeitssituation zu betrachten. Ziel der vorliegenden Studie war es, Zeitdaten der ärztlichen Tätigkeit in der Pneumologie zu erheben, um bestehende Arbeitsabläufe analysieren zu können.

Methode: Assistenzärzte wurden individuell über einen Zeitraum von 60 Arbeitstagen in einer pneumologischen Klinik begleitet. Dabei wurde ihre Tätigkeit sekundengenau über 565 Stunden mit einem Handcomputer aufgezeichnet.

Ergebnisse: Die Daten wiesen eine durchschnittliche Arbeitszeit von 9:15 Stunden nach $(\mathrm{SD}=0: 46: 59 \mathrm{~h})$. Unterschiedlich hohe Zeitanteile wurden für die indirekte und direkte Patientenversorgung, administrative Aufgaben und interne Kommunikation verwandt.

Diskussion: Die vorliegende Arbeit ist die erste Echtzeittätigkeitsanalyse im Bereich der Pneumologie. Auf Grundlage der vorliegenden Ergebnisse konnten die Angaben der Ärzte zum Teil bestätigt werden. Eine Reorganisation der bestehenden $\mathrm{Ar}$ beitsabläufe und der Einsatz von Assistenzsystemen könnte die wahrgenommene Arbeitsbelastung verringern.

\section{Einleitung}

$\nabla$

Das deutsche Gesundheitssystem ist derzeit durch Veränderungen bei der Finanzierung stationärer Versorgungsleistungen und der Festlegung des Leistungsumfangs der gesetzlichen Krankenversicherung gekennzeichnet [1]. Gegen-

\section{Abstract \\ $\nabla$}

Background: Currently, data from questionnaires have demonstrated increasing criticism from respiratory physicians regarding their working situation. They complain about long working hours, income and an increasing amount of time spent on documentation and administrative duties in particular. Since these criticisms are only subjective perceptions they should be compared to data that are more objective. Therefore, the aim of the current study was to collect precise data about physicians' work in respiratory medicine in order to analyse working routines.

Method: The working days of 60 physicians in a respiratory medicine hospital ward were observed unobtrusively for a total of 565 hours. An ultramobile personal computer was used to record the type and duration of all job tasks.

Results: The study results illustrate that a physician's working day amounted to an average of 9 hours 15 minutes ( $S D=0: 46: 59 \mathrm{~h}$ ). Within this time span different time periods were spent on administrative tasks, internal communication and meetings as well as on communication with patients, ward rounds and medical examinations. Conclusion: This is the first real-time analysis of job tasks in respiratory medical care. Some of the problems with work schedules reported by the doctors themselves were partly verified. Regarding the study results, a reorganisation of job tasks coupled with medical assistance systems may prove useful in reducing the workload of respiratory physicians.

wärtig müssen Krankenhäuser die rückläufigen finanziellen Mittel kompensieren. Dies wird über eine Erhöhung der Fallzahlen sowie durch eine Neugestaltung der ärztlichen Arbeitszeiten und Minderung der Personalkosten angestrebt. Durch die Einführung von Behandlungspfaden soll mittels Ablaufsoptimierung nicht nur die Behand- 
lungsqualität optimiert, sondern auch die Wirtschaftlichkeit verbessert werden. Für die Ärzte wird es jedoch immer schwieriger, die Patienten angemessen zu versorgen [1]: die dargestellten ökonomischen Rahmenbedingungen haben einen beträchtlichen Einfluss auf die ärztliche Tätigkeit, denn es wird zunehmend in das Aufgabengebiet des klinisch tätigen Arztes eingegriffen. Die uneingeschränkte Ausführung der erlernten ärztlichen Tätigkeit gegenüber dem Patienten stellt unter den gegenwärtigen Bedingungen eine Ausnahme dar [2]. Wieviel Zeit tatsächlich für nicht ärztliche Tätigkeiten (z.B. Verwaltungsaufgaben) verwendet wird, ist noch unklar. Aussagen existieren gegenwärtig nur aus subjektiven Einschätzungen.

Fragebogenstudien stellten die von Ärzten, darunter auch Pneumologen, wahrgenommenen Arbeitsbedingungen dar [3]. Hierbei wurden insbesondere eine enorme Arbeitsbelastung, die langen Arbeitszeiten und der hohe Anteil an administrativen Tätigkeiten als Kritikpunkte aufgezeigt [4-7].

Da es sich bei diesen Studien allein um subjektive Einschätzungen handelt und bisher keine objektiven Daten über die aktuelle Situation der ärztlichen Tätigkeit in der Pneumologie erhoben worden sind, ist das Ziel dieser Studie eine Tätigkeitsanalyse der Ärztinnen und Ärzte in der Pneumologie durchzuführen. Es sollen Aussagen über die derzeitige ärztliche Arbeitssituation in der stationären Versorgung mit objektiven Zeitdaten getroffen werden.

Diese Daten können die bereits aufgezeigten Angaben der Ärztinnen und Ärzte verifizieren oder falsifizieren. Zudem soll die Analyse einen wegweisenden Beitrag leisten, mögliche Verbesserungen in der Arbeitsgestaltung der Ärzte in der Pneumologie abzuleiten.

\section{Methodik \\ $\nabla$}

\section{Datenerhebung}

Die Datenerhebung fand zwischen Oktober 2008 und Februar 2009 in der pneumologischen Abteilung eines städtischen Krankenhaus (> 500 Betten) statt.

Zur Rekrutierung der Ärztinnen und Ärzte auf dieser Station wurde eine Informationsveranstaltung zur geplanten Studie durchgeführt. Nach der Einverständniserklärung der Klinikumsleitung wurden zwölf Assistenzärztinnen und -ärzte für die Studie rekrutiert.

Jeder der zwölf Ärzte wurde für fünf Arbeitstage über die gesamte Schicht begleitet. Während der Untersuchungszeit begleitete ein bereits mit der Methodik vertrauter Datenerheber den jeweiligen Arzt in einer Distanz von mindestens 2 Metern und zeichnete jede Tätigkeit des Arztes sekundengenau auf.

\section{Datenerfassungsprogramm}

Zunächst wurde ein speziell für diese Studie entwickeltes Datenprogramm entworfen [8], mit dessen Hilfe alle Tätigkeiten des Arztes sekundengenau aufgezeichnet werden konnten. Dieses Programm wurde dann auf einen tragbaren Handcomputer übertragen (UMPC, Samsung Q1, Samsung Inc., Schwalbach, Germany). Dreizehn Tätigkeitskategorien mit 50 Unterkategorien, die die Tätigkeiten eines Arztes in der Pneumologie umfassen, wurden als Vorgabe in das Programm integriert ( Tab. 1).

Der Datenerfasser codierte während der Begleitung jede Handlung des Arztes mit einer vorgegebenen Kategorie. Es wurde außerdem die zeitliche Dauer der jeweiligen Handlung aufgezeichnet.
Tab. 1 Tätigkeitskategorien.

\begin{tabular}{|c|c|}
\hline Kategorie & Tätigkeitsbeschreibung \\
\hline Visite & $\begin{array}{l}\text { Begutachtung des Patienten am Krankenbett } \\
\text { durch einen oder mehrere Ärzte }\end{array}$ \\
\hline $\begin{array}{l}\text { Aufnahme in das } \\
\text { Krankenhaus }\end{array}$ & $\begin{array}{l}\text { Hospitalisierung eines Erkrankten, Auf- } \\
\text { nahme der Krankengeschichte, erste Unter- } \\
\text { suchungen }\end{array}$ \\
\hline Pause & Erholungszeiten, Mittagspause \\
\hline Arztbrief & Schreiben des Arztbriefes \\
\hline Telefonate & Ärztliche Telefongespräche \\
\hline $\begin{array}{l}\text { Besprechungen und } \\
\text { interne Kommunikation }\end{array}$ & $\begin{array}{l}\text { Besprechungen mit Kollegen oder Vor- } \\
\text { gesetzten, Fort- und Weiterbildung }\end{array}$ \\
\hline $\begin{array}{l}\text { medizinische } \\
\text { Untersuchungen }\end{array}$ & $\begin{array}{l}\text { z. B. Bronchoskopie, Sonography, Thorax- } \\
\text { skopie, Blutentnahme, Infusionen }\end{array}$ \\
\hline Suchen & z. B. nach Dokumenten und Berichten suchen \\
\hline Warten & $\begin{array}{l}\text { auf Patienten, Kollegen oder Bereichte war- } \\
\text { ten, Warten wegen Computerproblemen }\end{array}$ \\
\hline Lesen & Fachliteratur, Röntgenbilder \\
\hline Weg & Wege im Krankenhaus \\
\hline Computerarbeit & schriftliche Dokumentation am Computer \\
\hline sonstige Dokumentation & $\begin{array}{l}\text { sämtliche schriftliche Dokumentation auf } \\
\text { Papier }\end{array}$ \\
\hline
\end{tabular}

\section{Datenanalyse}

Alle Tätigkeiten wurden in Echtzeit erhoben und nach vollständiger Erfassung in Excel (Microsoft Cooperation ${ }^{\circledR}$ ) analysiert und ausgewertet. Zusätzliche deskriptive Auswertungen wurden mit dem Datenanalyseprogramm SPSS ${ }^{\circledR} 15.0$ durchgeführt.

\section{Ergebnisse}

\section{Demographische Angaben der Ärztinnen und Ärzte}

Das Alter der untersuchten fünf Ärztinnen und sieben Ärzte variierte zwischen 26 und 36 Jahren. Das Durchschnittalter lag bei 32,63 Jahren ( $S D=3,28$ Jahre). Die durchschnittliche Berufserfahrenheit differierte zwischen 1 und 9 Jahren, durchschnittlich waren es 6,38 Jahre ( $\mathrm{SD}=3,12$ Jahre).

\section{Arbeitszeitanteile der Pneumologen}

Insgesamt wurden 60 Arbeitstage begleitet und alle in dieser Zeit ausgeführten Tätigkeiten aufgezeichnet.

Im Durchschnitt arbeiteten die Ärztinnen und Ärzte 9:15 Stunden $(95 \%$ CI 9:00:37 h bis 9:30:41 h). Innerhalb dieser Zeit verbrachten sie im Durchschnitt 49 Minuten für die Pausengestaltung (95\% CI 0:42:00 h bis 0:55:22 h). Die Länge der Arbeitstage variierte zwischen 8:01 Stunden (der kürzeste Arbeitstag) und 10:40 Stunden (der längste Arbeitstag).

Die größten Zeitanteile pro Beobachtungsperiode wurden für Besprechungen, Dokumentation und Administration sowie für Visite genutzt $(\bullet$ Abb. 1).

\section{Besprechungen und interne Kommunikation}

Insgesamt wurde der größte Tageszeitraum für Früh- und Mittagsbesprechungen verbracht $(\mathrm{M}=1: 40: 00 \mathrm{~h} ; 95 \% \mathrm{CI} 1: 28: 59 \mathrm{~h}$ bis $1: 51: 13$ h) ( Abb. 2).

Bis zu vier medizinische Besprechungen mit Kollegen (z.B. Ärzte, Pflegekräfte) wurden am Tag durchgeführt. Die durchschnittliche Kommunikationszeit mit Kollegen betrug 47 Minuten $(\mathrm{SD}=0: 22: 00 \mathrm{~h})$. Zeiten für Fort- und Weiterbildung umfassten 14 Minuten pro Tag (95\% CI 0:07:36 h bis 0:20:41 h). 


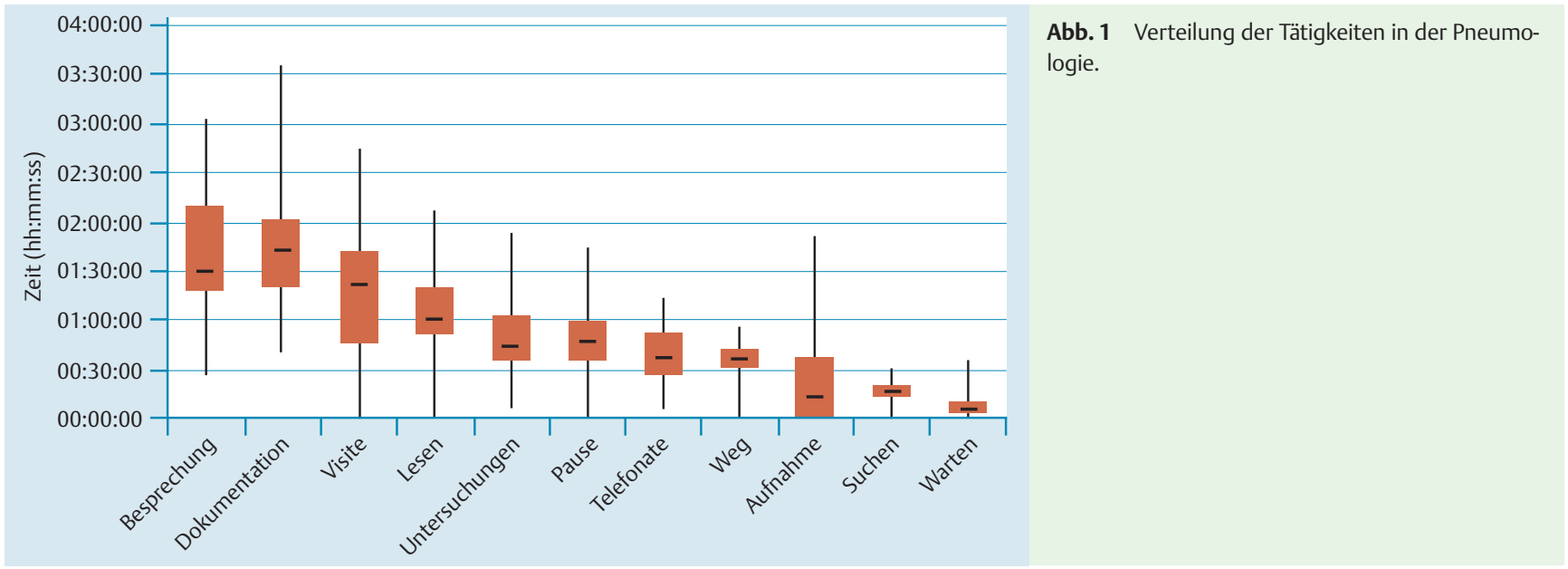

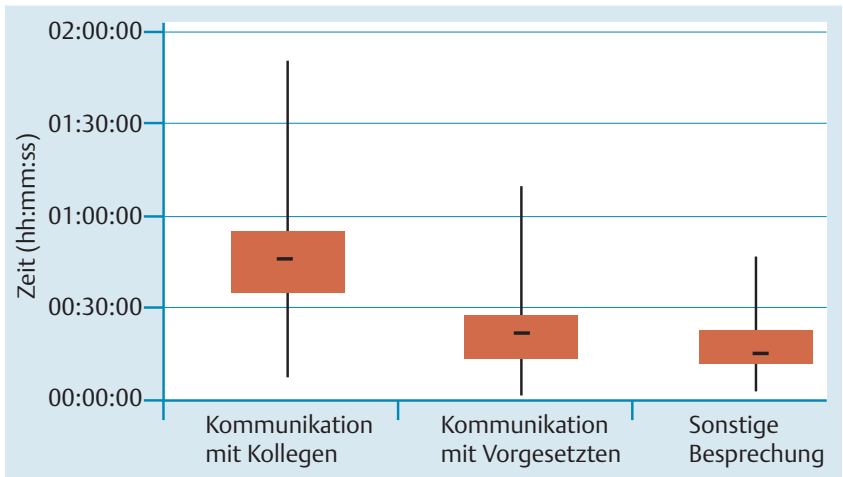

Abb. 2 Verteilung der Zeitanteile für Besprechungen und interne Kommunikation.

Patienten Management (Dokumentation,

Administration, Telefonate)

Im Durchschnitt wurden 18\% des Tages für Dokumentationsund Administrationsaufgaben verwendet $(\mathrm{M}=01: 41 \mathrm{~h} ; 95 \% \mathrm{CI}$ $1: 30: 59 \mathrm{~h}$ bis $1: 52: 25 \mathrm{~h}$ ). Diese Aufgaben beinhalteten: 1 . das Schreiben der Arztbriefe ( $M=0: 35: 26$ Minuten pro Tag; 95\% CI $0: 26: 37 \mathrm{~h}$ bis $0: 44: 14 \mathrm{~h}) ; 2$. die gesamte sonstige Dokumentation (bspw. das Verfassen von Berichten, sonstige klinische Notizen) $(\mathrm{M}=0: 28: 52 \mathrm{~h} ; 95 \% \mathrm{CI} 0: 25: 16 \mathrm{~h}$ bis $0: 32: 27 \mathrm{~h})$ und 3 . die Computerarbeit (z. B. Schreiben von E-Mails) (M = 0:37:24 h; $95 \% \mathrm{Cl} 0: 31: 13 \mathrm{~h}$ bis $0: 43: 34 \mathrm{~h}$ ).

38 Minuten 13 Sekunden $(95 \% \mathrm{Cl} 0: 32: 12 \mathrm{~h}$ bis $0: 44: 14 \mathrm{~h}$ ) wurden für Telefonate (z. B. Anforderung von Berichten und Untersuchungen) genutzt; das sind 6,8\% der täglichen Arbeitszeit. Die aufgezeichnete Zeit für das DRG-Codieren umfasste im Durchschnitt 4 Minuten 5 Sekunden pro Arzt und Arbeitstag $(95 \% \mathrm{CI}$ $0: 02: 22 \mathrm{~h}$ bis $0: 05: 48 \mathrm{~h}$ ).

\section{Medizinische Untersuchungen}

Untersuchungen am Patienten umfassten einen Zeitanteil von durchschnittlich 0:46:40 Minuten $(95 \%$ CI $0: 39: 27 \mathrm{~h}$ bis $0: 53: 52 \mathrm{~h}$ ) am Tag. Im Durchschnitt wurden 17 Patienten am Tag behandelt $(\mathrm{SD}=1,57)$.

Die medizinischen Untersuchungen in der Pneumologie bezogen sich u.a. auf die Sonografie, Bronchoskopie und Thoraxskopie. Die Blutentnahme beim Patienten wird durchschnittlich $0: 06: 49$ Minuten $(95 \% \mathrm{Cl} 0: 04: 52 \mathrm{~h}$ bis $0: 08: 47 \mathrm{~h}$ ) am Tag ausgeführt.

\section{Visite, Aufnahme und sonstige Arzt-Patienten-} Kommunikation

Die untersuchten Ärzte verbrachten 1:15:33 Stunden $(95 \%$ CI $1: 02: 28 \mathrm{~h}$ bis $1: 28: 38 \mathrm{~h}$ ) bei der täglichen Visite. Die Aufnahme von neuen Patienten ins Krankenhaus dauerte im Durchschnitt $0: 22: 23$ Minuten $(95 \% \mathrm{Cl} 0: 13: 33 \mathrm{~h}$ bis $0: 31: 14 \mathrm{~h}$ ).

Zusätzliche Zeitanteile für diagnostische und therapeutische Konversationen zwischen Arzt und Patient sowie für Aufklärungsgespräche betrugen am Tag durchschnittlich 1:16:17 Stunden (95\% Cl 1:09:05 h bis 1:23:30 h) ( $\bullet$ Abb. 3).

Insgesamt verbrachte ein Arzt durchschnittlich 0:06:44 Minuten der Arbeitszeit mit Angehörigengesprächen (95\% CI 0:04:00 h bis 0:09:09h). Diese Tätigkeit umfasste demgemäß $1,2 \%$ des Arbeitstages.

\section{Sonstige Zeitanteile}

Die begleiteten Ärzte in der Pneumologie verbrachten täglich im Durchschnitt 34 Minuten 13 Sekunden für Wege im Krankenhaus (95\% Cl $0: 30: 50 \mathrm{~h}$ bis $0: 37: 35 \mathrm{~h})$.

Arbeitshindernisse wie z.B. Warten auf Berichte, Patienten, Kollegen, Computerprobleme oder Suchen kosteten den Arzt im Durchschnitt 23 Minuten 34 Sekunden (95\% CI 0:19:54 h bis $0: 27: 13 \mathrm{~h}$ ). Die Zeitanteile für Warten betrugen im Durchschnitt $1,4 \%(\mathrm{M}=0: 07: 54 \mathrm{~h} ; 95 \% \mathrm{CI} 0: 05: 01 \mathrm{~h}$ bis $0: 10: 48 \mathrm{~h})$, für $\mathrm{Su}-$ chen $2,8 \%(\mathrm{M}=0: 15: 39 \mathrm{~h} ; 95 \% \mathrm{Cl} 0: 13: 47 \mathrm{~h}$ bis $0: 17: 31 \mathrm{~h})$.

\section{Diskussion}

$\checkmark$

Die vorliegende Studie ist die erste Echtzeitanalyse, die Aussagen über die ärztliche Tätigkeit in der Pneumologie trifft.

Die Ergebnisse zeigten, dass die durchschnittliche Arbeitswoche auf einer pneumologischen Station 46 Stunden umfasste. Unter Einbezug der Pausenzeiten mindert sich die Arbeitsstundenanzahl. Es zeigt sich, dass die Ärztinnen und Ärzte nur einen sehr geringen Überstundensatz leisten mussten.

Bezogen auf den gegenwärtig vorliegenden geringen Anteil an Überstunden, zeigt sich demnach eine Verbesserung hinsichtlich früherer Untersuchungen, in denen von bis zu 80 Arbeitsstunden in der Woche berichtet worden ist [9-11]. Diese Daten reflektieren sehr wahrscheinlich die Veränderungen im Arbeitszeitgesetz von 2007. Dementsprechend sei auch auf die relativ hohen Zeitanteile für Pausen hingewiesen. Ein Ergebnis, das aufgrund vorhergehender Angaben der Ärzte überrascht [12]. 


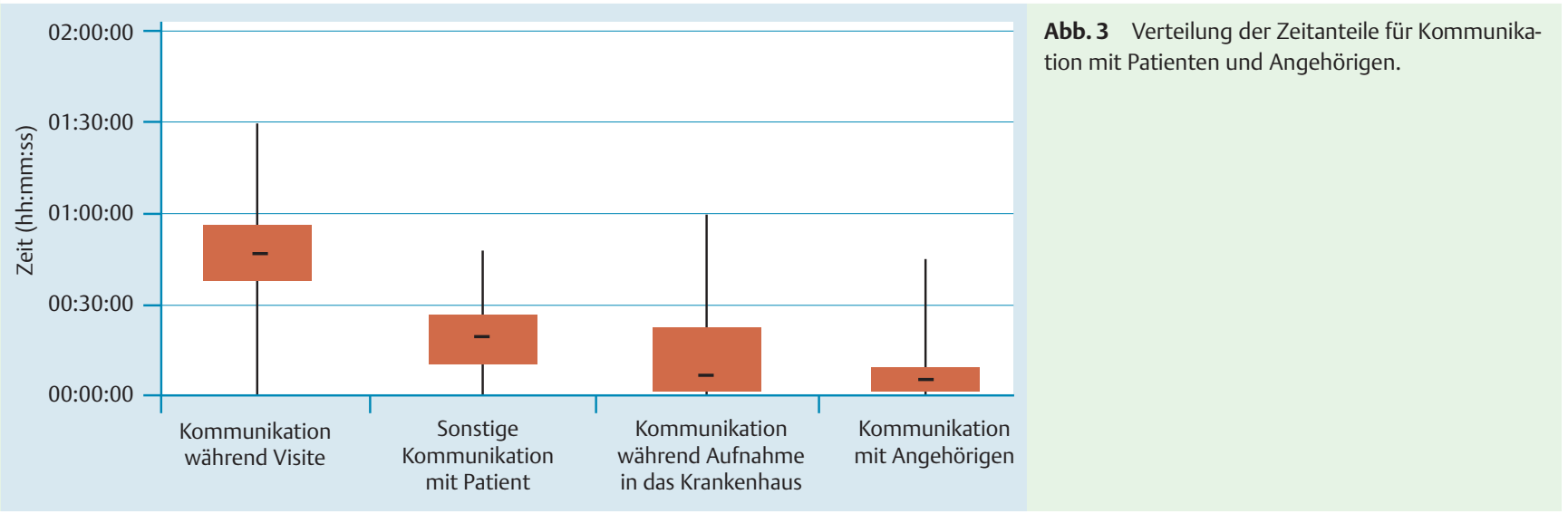

Es muss jedoch klar konstatiert werden, dass die externe Validität dieser Ergebnisse begrenzt ist, da die ärztliche Tätigkeit nur in einer Klinik untersucht worden ist. Somit müssen weitere Studien durchgeführt werden, die zeigen, dass die vorliegenden Ergebnisse nicht nur aufgrund einer in dieser Klinik spezifischen Situation zu begründen sind.

Die von Ärzten oft kritisierte Ausführung von Administrationsund Dokumentationstätigkeiten stellte sich in dieser Untersuchung als ein dominanter Zeitanteil dar. Dieses Ergebnis lässt sich durch 1) die zunehmenden Anforderungen an Dokumentations- und Administrationsvorgaben sowie 2) die steigende Anzahl Patienten pro Arzt begründen [13,14]. Studien führen diese Situation auch auf die DRG-Einführung zurück (Diagnosis Related Groups) [15,16], wenngleich gegenüberzustellen ist, dass die Zeitanteile für die DRG-Codierung in der vorliegenden Untersuchung gering ausfielen.

Eine Lösungsmöglichkeit, um die täglichen Arbeitsabläufe zu verbessern, könnte in der Reorganisation ärztlicher Tätigkeiten liegen. In diesem Sinne könnten Dokumentationsaufgaben und zu erledigende Telefonate teilweise in die Verantwortung eines Verwaltungsassistenten gegeben werden.

Der Einsatz eines solchen Assistenten würde den Zeitanteil für die direkte Patientenbehandlung erhöhen [17]. Zudem können diese Zeitanteile durch eine Verkürzung von Besprechungen, Wegen in der Klinik und sonstigen Arbeitshindernissen erhöht werden.

Da die vorliegende Studie einigen Einschränkungen unterliegt, können die gewonnenen Ergebnisse nicht bedingungslos verallgemeinert werden. Zunächst muss betont werden, dass die gewählte Methodik der Zeiterhebung das Arbeitsverhalten der Ärztinnen und Ärzte beeinflusst haben könnte (Hawthorne-Effekt). Es zeigte sich jedoch, dass die Ärzte aufgrund der langen Untersuchungszeit den Beobachter zeitweise gar nicht nicht mehr wahrgenommen haben bzw. ein tagelanger vorsätzlicher Bias des eigenen Zeitaufwandes für Tätigkeiten sehr belastend ist. Somit kann davon ausgegangen werden, dass es bei einer Wiederholung der Studie möglicherweise minimale Abweichungen in den Zeitwerten gibt, jedoch allgemeine Schlussfolgerungen aus den Ergebnissen nicht davon beeinflusst sind. Weitere Forschungsarbeiten in anderen Kliniken sind zwingend notwendig, um die vorliegenden Ergebnisse umfassend einordnen zu können.

Zudem sollten zukünftige Studien auf Wochenendarbeiten, Nachtschichten und saisonale Unterschiede ausgeweitet werden sowie eine umfangreichere Stichprobengröße einbeziehen.

\section{Schlussfolgerung}

Die vorliegende Studie ist die erste präzise Echtzeitanalyse, die einen Einblick in die Tätigkeit von Ärztinnen und Ärzten in der Pneumologie gibt. Die Daten unterstützen teilweise die kritischen Angaben der Ärzte über ihre wahrgenommenen Arbeitsbedingungen. Eine Reorganisation der Arbeitsabläufe verbunden mit einer Arbeitsaufteilung und dem Einsatz eines elektronischen Unterstützungssystems könnte die wahrgenommene Arbeitsbelastung der Ärzte verbessern, die Effektivität der Arbeit steigern und somit eine verbesserte Patientenversorgung gewährleisten.

\section{Interessenkonflikte \\ $\nabla$}

D. A. Groneberg - kein Interessenkonflikt

S. Mache - kein Interessenkonflikt

N. Jankowiak - kein Interessenkonflikt

C. Scutaru - kein Interessenkonflikt

\section{Literatur}

1 Hess R. One year of the German Health System Modernization Act and its impact on quality in medicine from the Joint Federal Committee's point of view. Z Arztl Fortbild Qualitatssich 2006; 100: 45-50

2 Peter S, Ulich E. Analyse der Arbeitssituation von Assistenz- und Oberärztinnen und -ärzten: Erfahrungen aus zwei Projekten. In: Ulich E, ed Arbeitspsychologie in Krankenhaus und Arztpraxis Arbeitsbedingungen, Belastungen, Ressourcen. Bern: Hans Huber, 2003: 75-98

3 Schwenzer KJ, Wang L. Assessing moral distress in respiratory care practitioners. Crit Care 2006; 34: 2967-2973

4 Huppertz H-I, Heckmann V, Marg W. Schichtdienst im Krankenhaus: Chancen und Probleme. Dtsch Arztebl 2004; 101: 324- 325

5 Arora VM, Georgitis E, Siddique J et al. Association of workload of oncall medical interns with on-call sleep duration, shift duration, and participation in educational activities. JAMA 2008; 300: 1146 - 1153

6 Mitchell J, Hayhurst C, Robinson SM. Can a senior house officer's time be used more effectively? Emerg Med 2004; 21: 545 - 547

7 Korzilius H. Zu den Auswirkungen der DRG-Einführung auf die Patienten und die Beschäftigten in den Kliniken. Dtsch Ärztebl 2006; 44: $2508-2513$

8 Mache S, Scutaru C, Vitzthum K et al. Development and evaluation of a computer-based medical work assessment programme. J Occup Med Toxicol (London, England) 2008; 3: 35

9 Jagsi $R$, Surender $R$. Regulation of junior doctors' work hours: an analysis of British and American doctors' experiences and attitudes. Soc Sci Med 2004; 58: 2181 - 2191

10 Jones $A M$, Jones $K B$. The 88 -hour family: effects of the 80 -hour work week on marriage and childbirth in a surgical residency. Iowa Orthop J 2007; 27: $128-133$ 
11 Pounder R. Junior doctors' working hours: can 56 go into 48? Clin Med (London, England) 2008; 8: 126-127

12 Ramsay MA. Physician fatigue. Proc (Bayl Univ Med Cent) 2000; 13: $148-150$

13 Deitmer T. Influence of working time regulations on the organisation and quality of clinical workflow. Z Arztl Fortbild Qualitatssich 2004; 98: 210 - 213 [discussion 213-215]

14 Janus K, Amelung VE, Gaitanides M, Schwartz FW. German physicians "on strike" - shedding light on the roots of physician dissatisfaction. Health policy (Amsterdam, Netherlands) 2007; 82: 357-365
15 Pelzer M, Diekmann D, Ryssel H, Germann G. Electronic documentation of injuries of the hand with a semantic network: effective and efficient methods for the documentation of clinical and administrative processes. Unfallchirurg 2007; 110: 213-218

16 Richburg BK. 5 nuances of Medicare billing. Healthc Financ Manage 2008; $62: 72-76$

17 Middeke M. Du bist Arzt in Deutschland. Dtsch Med Wochenschr 2005; 130: $2935-2936$

\section{Buchbesprechung}

Bewertung und Begutachtung in der Pneumologie

Nowak D, Kroidl RF (Hrsg)

3. und vollst. überarb. Aufl., Georg Thieme Verlag

Stuttgart 2009. 99,95€

ISBN 978-3-13-100 843-5

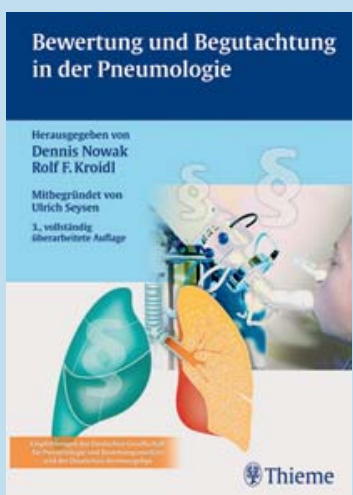

Wenn ein medizinisches Fachbuch seine 3. Auflage erlebt, besteht offensichtlich auf dem Markt eine große Bedürftigkeit nach den in ihm enthaltenen Informationen. Zweifels ohne trifft diese Annahme auf die neue Ausgabe des Begutachtungsbuches von Dennis Nowak und Rolf Kroidl zu.

Die 1. Auflage erfolgte 1995, seit dieser Zeit gehört das Buch in pneumologischen Kreisen zu einem regelrechten Bestseller. Das in jeder Hinsicht praxisgerechte Buch ist erweitert worden um Aspekte der Spiroergometrie, die Adaptation an die aktuelle Rechtssprechung, neue wissenschaftlich beschriebene pulmonale Noxen wie Passivrauch, Beryllium und Cadmium, die internationale Klassifikation der Funktionen, schlafbezogene Atmungsstörungen, neue bildgebende Verfahren sowie Stellungnahmen zur Beurteilung der Tauch- und Flugfähigkeit. Insgesamt 14 Autoren haben hierzu in 9 größeren Kapiteln Beiträge geleistet.

Die Kapitel 1 und 2 liefern die juristischen und gesetzlichen sowie verordnungsgebundenen Grundlagen, auf denen jede medizinische Bewertung und Begutachtung erfolgen muss. Das 3. Kapitel stellt ausführlich die technischen Grundlagen derjenigen Verfahren vor, die in ein Begutachtungsverfahren eingehen.

Kernpunkt des Buches ist schließlich das 4. Kapitel, das sehr ausführlich zur Bewertung einzelner pneumologisch-relevanter Krankheitsbilder Stellung nimmt (obstruktive Atem- wegserkrankung, Infektionskrankheiten, Lungengerüstkrankheiten, Tumorerkrankungen, schlafbezogene Atmungsstörungen und thorakale Defektzustände). Nahezu jeder sich in einem Gutachtenverfahren ergebenden Fragestellung wird dieses Buch gerecht, der Autor dieser Buchbesprechung hat dies anhand der letzten 3 erstellten Gutachten exemplarisch überprüft.

Von besonderem Wert ist sicherlich auch der Abschnitt über häufige Fehler bei der Begutachtung. Durch die Betrachtung dieser Problematik gewinnt die Qualität pneumologischer Gutachten ganz erheblich dazu.

Von Interesse für deutschsprachige, jedoch nicht in der Bundesrepublik Deutschland lebenden Kolleginnen und Kollegen sind die Abschnitte über das ärztliche Begutachtungsverfahren in der Schweiz und in Österreich. Wichtig für spezifische Fragestellungen, die sich aus der früheren deutschen Teilung ergeben, ist der Abschnitt über das Begutachtungswesen in der ehemaligen DDR.

Ebenfalls interessant sind die Ausführungen zur Tauchtauglichkeit und Flugtauglichkeit sowie der kurze Absatz über die Fragestellung Bergsteigen mit pulmonalen Erkrankungen. In der Summe eröffnet das auch sehr gut mit Literatur versehene Buch all denjenigen, die Begutachtungen durchführen, eine profunde Grundlage. Viele Fragestellungen lassen sich mit dem Büchlein exzellent beantworten. Als Referenz für pneumologische Gutachten sollte das Werk daher stets herangezogen werden. Es sei daher den Herausgebern und Autoren herzlich dafür gedankt, das ungemein praktische und nahezu unverzichtbare Kompendium aktualisiert zu haben.

Tom Schaberg, Rotenburg 